\title{
Energetic Stability of Coreless Vortices in Spin-1 Bose-Einstein Condensates with Conserved Magnetization
}

\begin{abstract}
Justin Lovegrove, Magnus O. Borgh, and Janne Ruostekoski
Mathematical Sciences, University of Southampton, SO17 1BJ Southampton, United Kingdom

(Received 19 June 2013; revised manuscript received 16 January 2014; published 19 February 2014)

We show that conservation of longitudinal magnetization in a spinor condensate provides a stabilizing mechanism for a coreless vortex phase-imprinted on a polar condensate. The stable vortex can form a composite topological defect with distinct small- and large-distance topology: the inner ferromagnetic coreless vortex continuously deforms toward an outer singular, singly quantized polar vortex. A similar mechanism can also stabilize a nonsingular nematic texture in the polar phase. A weak magnetization is shown to destabilize a coreless vortex in the ferromagnetic phase.
\end{abstract}

DOI: 10.1103/PhysRevLett.112.075301

In ordinary superfluids, quantization of circulation around a singular core is a hallmark of superfluidity. In superfluids with internal degrees of freedom, circulation need not be quantized, and it becomes possible for angular momentum to be carried by nonsingular textures. The canonical examples are the Anderson-ToulouseChechetkin (ATC) [1,2] and Mermin-Ho (MH) [3] vortices in superfluid liquid ${ }^{3} \mathrm{He}$. Coreless spin textures are also well known in quantum Hall physics [4].

Coreless vortices naturally occur in the ferromagnetic (FM) phase of the atomic spin-1 Bose-Einstein condensate (BEC), where the order parameter is determined by spatial spin rotations and circulation alone is not quantized $[5,6]$. Angular momentum is then carried by a characteristic, nonsingular fountainlike spin texture [7-11]. In the polar phase, by contrast, the expectation value of the spin vanishes, vortices are singular, and circulation is quantized $[5,6]$.

However, $s$-wave interactions between spin-1 atoms cannot change the longitudinal BEC magnetization $M=f_{+}-f_{-}$, where $f_{ \pm}$denote the fraction of atom population in levels $|m= \pm 1\rangle$. Experiments have demonstrated that $M$ is approximately conserved on the time scales of current alkali-metal atom experiments [12-14] and its value can be controlled. Constrained magnetization can force nonvanishing spin profiles to exist even in the polar regime. In strongly magnetized BECs, coreless vortices have indeed been prepared by phase-imprinting in the polar interaction regime [15-17] where nonsingular vortices would not be expected to appear by simple energetic arguments alone.

Here we study coreless, nonsingular vortex textures of spin-1 BECs in the general case where the system is no longer confined to either the FM or polar ground-state manifold. In particular, in the polar regime we show that the coreless vortex, formed by the spin texture, can be energetically stable in a rotating trap for sufficiently strong magnetization. In the numerics, the stability of imprinted
PACS numbers: 67.85.-d, 03.75.Lm, 03.75.Mn, 11.27.+d

coreless textures is studied by strictly constraining $M$ throughout the energy-relaxation process. We analyze the textures by constructing analytic models of their spinor wave functions that interpolate between the two manifolds. In the stable coreless polar vortex, the magnetization leads to a mixing of the polar and FM phases with a hierarchical core structure: The interior core region $\rho=$ $\left(x^{2}+y^{2}\right)^{1 / 2} \lesssim \eta_{M}$, where $\eta_{M}$ is the characteristic length scale determined by the magnetization constraint [18], exhibits a coreless vortex analogous to the coreless vortex in the FM phase. In the outer region, $\rho \gtrsim \eta_{M}$, the coreless vortex continuously deforms toward a singly quantized, singular polar vortex. With distinct small- and largedistance topology, the vortex represents a composite topological defect. Composite defects also exist, e.g., in high-energy physics and superfluid liquid ${ }^{3} \mathrm{He}$. Spinor BECs may therefore be able to shed light on generic topological features of composite vortex cores, acting as laboratory emulators [19]. However, there are also important distinct features: in atomic systems the physical origin of the composite core is different and results from a conservation law (for $M$ ). For instance, in ${ }^{3} \mathrm{He}$ the hierarchy of different core structures emerges from the interplay between different interaction energies, owing, e.g., to spin-orbit or magnetic-field coupling $[20,21]$.

The vortex core can also be understood in terms of characteristic energetic length scales. In a spin-1 BEC, where the magnetization is not conserved, the core size is determined by the larger of the two healing lengths $\xi_{F}$ or $\xi_{n}$ [22-24]. These describe, respectively, the distance over which local perturbations of the spin expectation value or the density heal [18]. When the conserved magnetization yields $\eta_{M} \gtrsim \max \left(\xi_{F}, \xi_{n}\right)$, it becomes energetically favorable to form a core of size $\eta_{M}$, with sharply varying magnetization density.

The phase-imprinting technique employed to create the coreless vortex with the fountainlike spin texture in 
Refs. $[15,16]$ can also be used to prepare a nematic coreless vortex (NCV) [16,17] in the polar regime, in which case the characteristic texture is formed by the nematic axis. The NCV does not carry angular momentum, and therefore cannot be stabilized by rotation. We show that a sufficiently strong magnetization-which empties one of the spinor components-however, can energetically stabilize the NCV. The stable configuration may also be understood in terms of a composite vortex: in the inner region the nematic axis forms a fountainlike coreless texture that continuously deforms in the outer region to a singular, singly quantized FM vortex. The spin profile then provides a constraint, stabilizing the NCV inside the core.

Substantial deviations of the conserved magnetization from its "unconstrained" value (i.e., when magnetization is allowed to relax) can also affect the FM regime. We show that a singly quantized FM vortex can form the ground state in a rotating condensate. This may seem surprising, since in that case coreless vortices are otherwise predicted to make up the ground state [8-11]. We find that the spin structure of the coreless vortex cannot support a weak magnetization and the singly quantized singular vortex, which would normally exhibit higher energy than the coreless vortex [23], becomes the new ground state.

We consider the classical mean-field theory of the spin-1 $\mathrm{BEC}$, with the wave function given by the atom density $n(\mathbf{r})$ and a three-component spinor $\zeta(\mathbf{r})$,

$$
\Psi(\mathbf{r})=\sqrt{n(\mathbf{r})} \zeta(\mathbf{r})=\sqrt{n(\mathbf{r})}\left(\begin{array}{c}
\zeta_{+}(\mathbf{r}) \\
\zeta_{0}(\mathbf{r}) \\
\zeta_{-}(\mathbf{r})
\end{array}\right), \quad \zeta^{\dagger} \zeta=1 .
$$

The $s$-wave interaction between two spin- 1 atoms, with the scattering lengths $a_{0}$ and $a_{2}$, acquires a spin-dependent contribution, yielding two interaction terms $\left[c_{0}=4 \pi \hbar^{2}\right.$ $\left(2 a_{2}+a_{0}\right) / 3 m$ and $\left.c_{2}=4 \pi \hbar^{2}\left(a_{2}-a_{0}\right) / 3 m\right]$ in the Hamiltonian density [18],

$$
\mathcal{H}=\frac{\hbar^{2}}{2 m}|\nabla \Psi|^{2}+\frac{1}{2} m \omega^{2} r^{2} n+\frac{c_{0}}{2} n^{2}+\frac{c_{2}}{2} n^{2}|\langle\hat{\mathbf{F}}\rangle|^{2},
$$

where $\omega$ is the trap frequency, and $\hat{\mathbf{F}}$ the spin operator, such that $\langle\hat{\mathbf{F}}\rangle=\zeta_{\alpha}^{\dagger} \hat{\mathbf{F}}_{\alpha \beta} \zeta_{\beta}$ yields the local spin vector.

The sign of $c_{2}$ in Eq. (2) determines which phase is energetically favored by the spin-dependent interaction alone. If $c_{2}>0$, as with ${ }^{23} \mathrm{Na}$, the polar phase with $|\langle\hat{\mathbf{F}}\rangle|=0$ is preferred. The order parameter is then defined by a BEC phase $\phi(\mathbf{r})$ and an unoriented nematic axis $\hat{\mathbf{d}}(\mathbf{r})$ $[5,6]$. The two choices $(\phi, \hat{\mathbf{d}})$ and $(\phi+\pi,-\hat{\mathbf{d}})$ represent the same physical state and must therefore be identified. This nematic order leads to the existence of vortices with both integer and half-integer [25] winding.

Conversely if $c_{2}<0$, as with ${ }^{87} \mathrm{Rb}$, the $\mathrm{FM}$ phase with $|\langle\hat{\mathbf{F}}\rangle|=1$ is favored. All FM spinors are related by three-dimensional spin rotations, which define the orderparameter space. This supports two classes of line defects $[7,26]$. The nontrivial representatives of each class are singular, singly quantized vortices and nonsingular, coreless vortices, respectively.

The rich phenomenology of the spin-1, and higher-spin, order-parameter spaces provides an intriguing system for studies of defects and textures [5,7-11,22-44]. Experimentally, vortices and spin textures have been investigated by means of both controlled preparation [15-17,45] and dynamical formation [46-49]. Here we consider the energetic stability of a coreless vortex when the magnetization is conserved. The coreless vortex may be phaseimprinted onto a BEC with initially uniform spin texture, regardless of the sign of $c_{2}$. The imprinted vortex state then relaxes due to energy dissipation and we investigate its structure and stability. The magnetization constraint may in general force the wave function out of its ground-state manifold by mixing the FM and polar phases. We will show that the energy relaxation of the prepared vortex can then be analyzed by introducing the spinor wave function

$$
\zeta^{\mathrm{cl}}(\mathbf{r})=\frac{1}{2}\left(\begin{array}{c}
\sqrt{2}\left(D_{-} \sin ^{2} \frac{\beta}{2}-D_{+} \cos ^{2} \frac{\beta}{2}\right) \\
-e^{i \varphi}\left(D_{-}+D_{+}\right) \sin \beta \\
\sqrt{2} e^{2 i \varphi}\left(D_{-} \cos ^{2} \frac{\beta}{2}-D_{+} \sin ^{2} \frac{\beta}{2}\right)
\end{array}\right) .
$$

Here $D_{ \pm}=\sqrt{1 \pm F}$, so that in cylindrical coordinates $(\rho$, $\varphi, z)$ the spin vector $\langle\hat{\mathbf{F}}\rangle=F(\sin \beta \hat{\boldsymbol{\rho}}+\cos \beta \hat{\mathbf{z}})$, with $|\langle\hat{\mathbf{F}}\rangle|=F$, displaying the characteristic fountainlike texture. The coreless vortex (3) interpolates between the FM and polar phases with $0 \leq F \leq 1$. It can be constructed from a generalized spinor by combining a $2 \pi$ winding of the BEC phase with a spin rotation [18].

In the limit $F=1$, the spinor (3) represents the ordinary coreless vortex solution in the FM phase, with the spin orientation $\beta(\rho)$ increasing monotonically from zero at $\rho=0$. In the opposite limit $F=0$, it becomes a singly quantized polar vortex, with $\hat{\mathbf{d}}=\cos \beta \hat{\boldsymbol{\rho}}-\sin \beta \hat{\mathbf{z}}$, displaying a radial disgyration for $\beta \neq \pi / 2$ [18]. Now we let $F$ vary in space such that $F(\rho \rightarrow 0) \rightarrow 1$ and $F \rightarrow 0$ for large $\rho$. Equation (3) then describes a composite vortex, where the inner coreless vortex forms the core of the outer polar vortex, and the circulation $\nu=\oint d \mathbf{r} \cdot \mathbf{v}=(\mathrm{h} / \mathrm{m})$ $(1-F \cos \beta)$, where $\mathbf{v}=(\hbar / 2 i m)\left[\zeta^{\dagger}(\nabla \zeta)-\left(\nabla \zeta^{\dagger}\right) \zeta\right]$ is the superfluid velocity, interpolates smoothly between the corresponding regions.

The coreless vortex is analogous to the nonsingular ATC $[1,2]$ and $\mathrm{MH}[3]$ textures in superfluid liquid ${ }^{3} \mathrm{He}$. For the coreless texture, one may define a winding number similar to that of a point defect [18],

$$
W=\frac{1}{8 \pi} \int_{\mathcal{S}} \mathrm{d} \Omega_{i} \epsilon_{i j k} \hat{\mathbf{n}}_{F} \cdot\left(\frac{\partial \hat{\mathbf{n}}_{F}}{\partial x_{j}} \times \frac{\partial \hat{\mathbf{n}}_{F}}{\partial x_{k}}\right),
$$

where $\hat{\mathbf{n}}_{F}=\langle\hat{\mathbf{F}}\rangle /|\langle\hat{\mathbf{F}}\rangle|$ and $\mathcal{S}$ denotes a surface covering the full cross section of the vortex core. The charge $W$ counts the number of times $\langle\hat{\mathbf{F}}\rangle$ wraps around the unit sphere. If the spin vector reaches a uniform asymptotic orientation everywhere away from the vortex (i.e., the bending angle $\beta(\rho)$ is an integer multiple of $\pi$ ), $W$ represents an integer-valued 
charge. This charge is conserved whenever the boundary condition is fixed, e.g., by physical interaction or energetics. If no boundary condition is imposed, the texture can unwind to the vortex-free state by purely local transformations of the wave function. The spin- 1 coreless vortex may be stabilized by rotation as the bending angle $\beta(\rho)$ in Eq. (3), and therefore the superfluid circulation, adapts to the imposed rotation. As a result of (4), coreless textures are also called two-dimensional "baby Skyrmions" [50] in analogy with the three-dimensional Skyrmions [51], which represent stable particlelike solitons that can also exist in atomic BECs [52-56].

Magnetic-field rotation [57-59] can be used to phaseimprint a coreless vortex $[15,16]$. In Ref. [16], the axial bias field $B_{z}$ of an external magnetic quadrupole field $\mathbf{B}=B^{\prime} \rho \hat{\boldsymbol{\rho}}+\left(B_{z}-2 B^{\prime} z\right) \hat{\mathbf{z}}$, is swept linearly such that the zero-field point passes through an initially spin-polarized BEC $\zeta^{1}=(1,0,0)^{T}[18]$. The sweep amounts to a spin rotation $\zeta^{\mathrm{i}}=\exp [-i \hat{\mathbf{F}} \cdot \beta(\rho) \hat{\boldsymbol{\varphi}}] \zeta^{1}$, resulting in the coreless vortex (3), with $F=1$ everywhere. A (pseudo)spin-1 coreless vortex has also been phase-imprinted in the $|m=0, \pm 2\rangle$ levels of a spin-2 ${ }^{87} \mathrm{Rb} \mathrm{BEC}$ (with the $|m= \pm 1\rangle$ levels empty) through population transfer using a Laguerre-Gaussian laser [18,45].

By adjusting the spin orientation $\beta(\rho)$ in imprinting experiments, one can accurately control the longitudinal magnetization $M$. As the imprinted texture relaxes, $M$ is then approximately conserved on time scales where $s$-wave scattering dominates, e.g., over dipolar interactions and collisions with high-temperature atoms [12-14,60]. Dynamical stability of a cylindrically symmetric, magnetized coreless vortex was demonstrated against low-energy, cylindrically symmetric Bogoliubov modes in Ref. [39].

We first study the energetic stability of the phaseimprinted coreless vortex in the polar regime. As an initial state we take the magnetically rotated $\zeta^{i}$ [Eq. (3) with $F=1$ everywhere], for different $M$. The energetic stability and structure of the vortex is then determined by numerically minimizing the free energy in a rotating trap (at the frequency $\Omega$ ). We take $N c_{0}=1000 \hbar \omega l^{3}$ and $c_{0} / c_{2} \simeq 28$ of ${ }^{23} \mathrm{Na}$ [61] in Eq. (2), where $l=(\hbar / m \omega)^{1 / 2}$ and $N$ is the number of atoms. We strictly conserve the longitudinal magnetization of the initial state throughout the relaxation procedure, by simultaneous renormalization of $M$ and $N$. The physical mechanism is therefore different from that used in numerical techniques to achieve a nonzero magnetization for the final state by means of an effective linear Zeeman term, without explicitly conserving the magnetization during relaxation $[8,28-30]$.

In a spin- 1 BEC a vortex singularity can be accommodated by exciting the wave function out of its ground-state manifold, whenever it is energetically more favorable to adjust the spin value than force the density to vanish at the singular core [22,23]. This happens when the characteristic length scales [18] $\xi_{n}=l\left(\hbar \omega / 2 c_{0} n\right)^{1 / 2}$ and $\xi_{F}=l\left(\hbar \omega / 2\left|c_{2}\right| n\right)^{1 / 2}$ satisfy $\xi_{F} \gtrsim \xi_{n}$. Conservation of magnetization introduces a third length scale $\eta_{M}$ that describes in the polar phase the size of a FM core needed to yield the required $M$. By considering a uniformly magnetized cylindrical core inside an otherwise polar density profile, we find by a straightforward integration the estimate $\eta_{M}=R_{\mathrm{TF}} \sqrt{1-(1-M)^{2 / 5}}$, where $R_{\mathrm{TF}}$ is the Thomas-Fermi radius [18].

As the energy of the imprinted coreless vortex relaxes, the outer region approaches the singly quantized vortex with $F \rightarrow 0$. In the limit of weak magnetization, the vortex core splits into two half-quantum vortices, similarly to the splitting of a singly quantized vortex when magnetization is not conserved [23]. At $M=0$, the fountain texture is lost entirely. When $M$ increases, the stable coreless-vortex spin texture gradually becomes more pronounced, preventing the core splitting. The vortex (Fig. 1, top) still maintains the axial asymmetry of the magnetized core region with two close-lying spin maxima $F=1$, and $\langle\hat{\mathbf{F}}\rangle \| \hat{\mathbf{z}}$ at the center. Ignoring the slight core asymmetry, the vortex can be qualitatively described by the analytic model (3): The spin winds to $\langle\hat{\mathbf{F}}\rangle \| \hat{\boldsymbol{\rho}}$ as $\rho$ increases. Simultaneously, $F$ decreases sharply and the configuration approaches a singly quantized polar vortex. The size of the core (the magnetizationdensity half width at half maximum) is $\sim \eta_{M}$. Comparison of length scales then suggests that the coreless texture becomes pronounced when $\eta_{M} \gtrsim \xi_{F}\left(>\xi_{n}\right)$, in qualitative agreement with our numerics.

Owing to the trap, $F$ can reach a local minimum — which may not vanish in all directions - and start increasing at the edge of the cloud. The vortex profile then depends on $M$ and, e.g., on the quadratic Zeeman shift that favors the
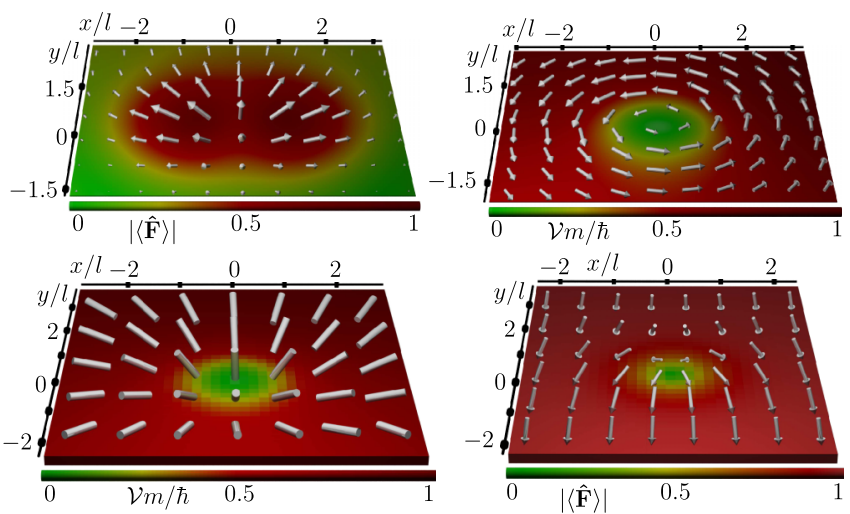

FIG. 1 (color online). Top left: Spin profile $\langle\hat{\mathbf{F}}\rangle$ (arrows, whose length together with the color gradient give $|\langle\hat{\mathbf{F}}\rangle|)$ of the energetically stable coreless vortex in the polar regime, interpolating between FM and polar phases and displaying the characteristic fountain texture inside the core of a singular polar vortex. Top right: The corresponding continuously varying superfluid velocity $\mathbf{v}$ and its magnitude (arrows), and circulation density $\mathcal{V}=\rho \mathbf{v} \cdot \hat{\boldsymbol{\varphi}}$ (color gradient) [18]. Bottom: Energetically stable $\mathrm{NCV}$, with fountain texture in $\hat{\mathbf{d}}$ (left, cylinders) together with $\mathcal{V}$ (color gradient), and corresponding $\langle\hat{\mathbf{F}}\rangle$ (right, arrows and color gradient). Conservation of magnetization forces the BEC into the FM phase away from the vortex line. 
polar phase [62]. We may therefore envisage a scheme to engineer the core symmetry and even more complex composite defects by Laguerre-Gaussian lasers that generate a Zeeman shift with a cylindrical shell symmetry.

For stronger magnetization we obtain an effective twocomponent coreless-vortex state, where $\zeta_{0}$ represents a singly quantized vortex whose core is filled by $\zeta_{+}$. The transition to the two-component system occurs when the $\beta(\rho)$ profile no longer allows the three-component vortex (3) to satisfy the magnetization constraint, depopulating $\zeta_{-}$. The threshold magnetization value decreases with rotation until at $\Omega \simeq 0.35 \omega$ the coreless vortex is stable only in the two-component regime (Fig. 2). For large $c_{0}$, the stable two-component solution represents a smooth transition from the $F=1$ coreless vortex at the trap center to the $F=0$ singular vortex at the edge of the cloud.

Next we study the stability of the coreless vortex with conserved magnetization in the FM regime using $N c_{0}=$ $1000 \hbar \omega l^{3}$ and $N c_{2} \simeq-5 \hbar \omega l^{3}$ of ${ }^{87} \mathrm{Rb}$ [63]. In this case the FM phase with $F=1$ is preserved everywhere in the imprinted spinor $\zeta^{i}$ during relaxation. We find that imposed weak magnetization can lead to energetic instability of the coreless vortex, with a singular vortex then becoming the rotating ground state. This may seem surprising, since when the magnetization is not conserved in energy relaxation, coreless vortices are predicted to form the ground state at sufficiently rapid rotation [8-11,23]; a singular vortex may then also be energetically (meta)stable, but always has a higher energy than the coreless vortex [23].

If magnetization is not conserved, its value for the stable coreless spin texture at the trap center is sensitive to rotation, e.g., varying in the studied case from $M \simeq 0.56$ at $\Omega \simeq 0.25 \omega$ to $M \simeq 0.34$ at $\Omega \simeq 0.3 \omega$. When the conservation of magnetization imposes weaker $M$ at a given rotation frequency, we find that the vortex is displaced from the trap center as it adjusts to the constraint. At very weak $M$ the coreless vortex is forced close to the edge of the cloud and splits into a pair of singular vortices, one of which exits the BEC. At faster rotation, the fountain profile bends more sharply, and consequently a smaller $M$ can be conserved in the coreless-vortex ground state (Fig. 2) [64].

The magnetic-field rotation technique used to phaseimprint the FM coreless vortex $[15,16]$ can also be applied to a BEC prepared in the polar state $\zeta^{0}=(0,1,0)^{T}[16,17]$,

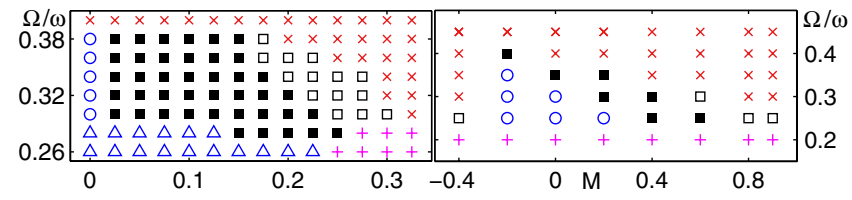

FIG. 2 (color online). Stability of the coreless vortex in the polar (left) and FM (right) interaction regimes: (filled square) stable coreless vortex; (empty square) stable effective twocomponent coreless vortex; instability towards (upward triangle) a half-quantum vortex, (circle) pair of half-quantum vortices (polar regime) or singular vortex (FM regime), (plus) vortex-free state; (cross) nucleation of additional vortices. in which the nematic axis $\hat{\mathbf{d}}=\hat{\mathbf{z}}$ and $M=0$. The rotation $\zeta^{\mathrm{p}}=\exp \left[-i \hat{\mathbf{F}} \cdot \beta^{\prime}(\rho) \hat{\boldsymbol{\varphi}}\right] \zeta^{0}[16]$ induced by the magneticfield sweep then leads to the nematic texture $\hat{\mathbf{d}}=$ $\sin \beta^{\prime} \hat{\boldsymbol{\rho}}+\cos \beta^{\prime} \hat{\mathbf{z}}$, which defines a nematic coreless vortex. Owing to the magnetic-field rotation, this always exhibits vanishing longitudinal magnetization. In Ref. [17] an ATClike (MH-like) texture was imprinted, with $\beta^{\prime}(\rho=0)=0$ and $\beta^{\prime}=\pi\left(\beta^{\prime}=\pi / 2\right)$ at the edge of the cloud. The winding number $W$ may be defined by taking $\hat{\mathbf{n}}_{F} \rightarrow \hat{\mathbf{d}}$ in Eq. (4). The value of $\hat{\mathbf{d}}$ is not fixed at the boundary, and the coreless nematic texture may smoothly dissolve. Unlike the spin texture of the ordinary coreless vortex, it cannot be stabilized by rotation, due to its vanishing mass circulation.

We ask instead whether the NCV can be stable inside the core of a composite topological defect when a conserved, nonzero $M$ necessitates formation of nonpolar regions. A NCV with a nonzero magnetization could be created by phase-imprinting via population transfer $[45,65,66]$ that individually prepares the appropriate phase windings of $\langle-2 \pi, 0,+2 \pi\rangle$ in the spinor components. In order to describe the composite core structure of the nematic coreless vortex we introduce the spinor wave function [18]

$$
\zeta^{\mathrm{n}}=\frac{1}{2}\left(\begin{array}{c}
\sqrt{2} e^{-i \varphi}\left(D_{+} \cos ^{2} \frac{\beta}{2}-D_{-} \sin ^{2} \frac{\beta}{2}\right) \\
\left(D_{+}+D_{-}\right) \sin \beta \\
\sqrt{2} e^{i \varphi}\left(D_{+} \sin ^{2} \frac{\beta}{2}-D_{-} \cos ^{2} \frac{\beta}{2}\right)
\end{array}\right),
$$

where $\langle\hat{\mathbf{F}}\rangle=F(\sin \beta \hat{\boldsymbol{\rho}}+\cos \beta \hat{\mathbf{z}})$. From $\hat{\mathbf{d}} \perp\langle\hat{\mathbf{F}}\rangle$, it follows that in order to have the fountain texture in $\hat{\mathbf{d}}$ we must have $\beta=\pi / 2$ at $\rho=0$ and increasing monotonically. Then when $F(\rho)$ increases with $\rho$ from $F=0$ to $F=1$, $\zeta^{\text {n }}$ interpolates smoothly from the interior NCV to an outer singular FM vortex with radial spin component $\langle\hat{\mathbf{F}}\rangle_{\rho}=\sin \beta$, with $\beta \simeq \pi$ at the edge of the cloud (MH-like texture). The corresponding mass circulation, $\oint d \mathbf{r} \cdot \mathbf{v}=-(h F / m) \cos \beta$, interpolates from the noncirculating polar core to a nonzero circulation, in principle allowing stabilization by rotation [18].

We find that the numerical relaxation process of the phase-imprinted $\langle-2 \pi, 0,+2 \pi\rangle$ vortex configuration at given $M$ can be qualitatively described by the spinor (5). For the energetically stable NCV (Fig. 1, bottom), however, $\zeta_{+}$is depopulated and $\zeta_{0}$ fills the vortex line in $\zeta_{-}$, with the fountain structure in $\hat{\mathbf{d}}$. The core size is again determined by the magnetization constraint [18].

In conclusion, we have shown that the conservation of magnetization in spinor BECs makes it possible to produce vortex core structures with distinct small- and largedistance topology, by mixing of different phases. The characteristic core size is then determined by the magnetization constraint, instead of one of the healing lengths. In particular, a nonsingular, coreless vortex with sufficiently strong magnetization is energetically stable in the polar regime where simple energetic arguments alone would not predict its existence. The stable configuration can be qualitatively understood by an analytic model for the 
spinor wave function that interpolates between the FM and polar phases, representing a composite topological defect.

We acknowledge financial support from the Leverhulme Trust and EPSRC.

[1] V. R. Chechetkin, Zh. Eksp. Teor. Fiz. 71, 1463 (1976) [JETP 44, 766 (1976)].

[2] P. W. Anderson and G. Toulouse, Phys. Rev. Lett. 38, 508 (1977).

[3] N. D. Mermin and T.-L. Ho, Phys. Rev. Lett. 36, 594 (1976).

[4] D.-H. Lee and C. L. Kane, Phys. Rev. Lett. 64, 1313 (1990).

[5] Y. Kawaguchi and M. Ueda, Phys. Rep. 520, 253 (2012).

[6] D. M. Stamper-Kurn and M. Ueda, arXiv:1205.1888 (2012).

[7] T.-L. Ho, Phys. Rev. Lett. 81, 742 (1998).

[8] T. Mizushima, K. Machida, and T. Kita, Phys. Rev. Lett. 89, 030401 (2002).

[9] J.-P. Martikainen, A. Collin, and K.-A. Suominen, Phys. Rev. A 66, 053604 (2002).

[10] J. W. Reijnders, F. J. M. van Lankvelt, K. Schoutens, and N. Read, Phys. Rev. A 69, 023612 (2004).

[11] E. J. Mueller, Phys. Rev. A 69, 033606 (2004).

[12] J. Stenger, S. Inouye, D. M. Stamper-Kurn, H. Miesner, A. P. Chikkatur, and W. Ketterle, Nature (London) 396, 345 (1998).

[13] M.-S. Chang, C. D. Hamley, M. D. Barrett, J. A. Sauer, K. M. Fortier, W. Zhang, L. You, and M. S. Chapman, Phys. Rev. Lett. 92, 140403 (2004).

[14] D. Jacob, L. Shao, V. Corre, T. Zibold, L. De Sarlo, E. Mimoun, J. Dalibard, and F. Gerbier, Phys. Rev. A 86, 061601 (2012).

[15] A. E. Leanhardt, Y. Shin, D. Kielpinski, D. E. Pritchard, and W. Ketterle, Phys. Rev. Lett. 90, 140403 (2003).

[16] J.-y. Choi, W. J. Kwon, M. Lee, H. Jeong, K. An, and Y.-i. Shin, New J. Phys. 14, 053013 (2012).

[17] J.-y. Choi, W. J. Kwon, and Y.-i. Shin, Phys. Rev. Lett. 108, 035301 (2012).

[18] See Supplemental Material at http://link.aps.org/ supplemental/10.1103/PhysRevLett.112.075301for further details on mean-field theory, conservation of magnetization, length scales, phase imprinting, and analytic vortex solutions.

[19] I. Bloch, J. Dalibard, and S. Nascimbene, Nat. Phys. 8, 267 (2012).

[20] Ü. Parts, J. M. Karimäki, J. H. Koivuniemi, M. Krusius, V. M. H. Ruutu, E. V. Thuneberg, and G. E. Volovik, Phys. Rev. Lett. 75, 3320 (1995).

[21] M. M. Salomaa and G. E. Volovik, Rev. Mod. Phys. 59, 533 (1987).

[22] J. Ruostekoski and J. R. Anglin, Phys. Rev. Lett. 91, 190402 (2003).

[23] J. Lovegrove, M. O. Borgh, and J. Ruostekoski, Phys. Rev. A 86, 013613 (2012).

[24] S. Kobayashi, Y. Kawaguchi, M. Nitta, and M. Ueda, Phys. Rev. A 86, 023612 (2012).

[25] U. Leonhardt and G. Volovik, JETP Lett. 72, 46 (2000).

[26] T. Ohmi and K. Machida, J. Phys. Soc. Jpn. 67, 1822 (1998).
[27] H. T. C. Stoof, E. Vliegen, and U. Al Khawaja, Phys. Rev. Lett. 87, 120407 (2001).

[28] S.-K. Yip, Phys. Rev. Lett. 83, 4677 (1999).

[29] T. Isoshima and K. Machida, Phys. Rev. A 66, 023602 (2002).

[30] T. Mizushima, K. Machida, and T. Kita, Phys. Rev. A 66, 053610 (2002).

[31] C. M. Savage and J. Ruostekoski, Phys. Rev. A 68, 043604 (2003).

[32] F. Zhou, Int. J. Mod. Phys. B 17, 2643 (2003).

[33] H. Saito, Y. Kawaguchi, and M. Ueda, Phys. Rev. Lett. 96, 065302 (2006).

[34] L. Santos and T. Pfau, Phys. Rev. Lett. 96, 190404 (2006).

[35] V. Pietilä, M. Möttönen, and S. M. M. Virtanen, Phys. Rev. A 76, 023610 (2007).

[36] G. W. Semenoff and F. Zhou, Phys. Rev. Lett. 98, 100401 (2007).

[37] R. Barnett, A. Turner, and E. Demler, Phys. Rev. A 76, 013605 (2007).

[38] A.-C. Ji, W. M. Liu, J. L. Song, and F. Zhou, Phys. Rev. Lett. 101, 010402 (2008).

[39] M. Takahashi, V. Pietilä, M. Möttönen, T. Mizushima, and K. Machida, Phys. Rev. A 79, 023618 (2009).

[40] J. A. M. Huhtamäki, T. P. Simula, M. Kobayashi, and K. Machida, Phys. Rev. A 80, 051601 (2009).

[41] M. Kobayashi, Y. Kawaguchi, M. Nitta, and M. Ueda, Phys. Rev. Lett. 103, 115301 (2009).

[42] T. P. Simula, J. A. M. Huhtamäki, M. Takahashi, T. Mizushima, and K. Machida, J. Phys. Soc. Jpn. 80, 013001 (2011).

[43] M. O. Borgh and J. Ruostekoski, Phys. Rev. Lett. 109, 015302 (2012).

[44] M. O. Borgh and J. Ruostekoski, Phys. Rev. A 87, 033617 (2013).

[45] L. S. Leslie, A. Hansen, K. C. Wright, B. M. Deutsch, and N. P. Bigelow, Phys. Rev. Lett. 103, 250401 (2009).

[46] L. E. Sadler, J. M. Higbie, S. R. Leslie, M. Vengalattore, and D. M. Stamper-Kurn, Nature (London) 443, 312 (2006).

[47] M. Vengalattore, S. R. Leslie, J. Guzman, and D. M. Stamper-Kurn, Phys. Rev. Lett. 100, 170403 (2008).

[48] J. Kronjäger, C. Becker, P. Soltan-Panahi, K. Bongs, and K. Sengstock, Phys. Rev. Lett. 105, 090402 (2010).

[49] E. M. Bookjans, A. Vinit, and C. Raman, Phys. Rev. Lett. 107, 195306 (2011).

[50] N. Manton and P. Sutcliffe, Topological Solitons (Cambridge University Press, Cambridge, England, 2004).

[51] T. H. R. Skyrme, Proc. R. Soc. A 260, 127 (1961).

[52] J. Ruostekoski and J. R. Anglin, Phys. Rev. Lett. 86, 3934 (2001).

[53] R. A. Battye, N. R. Cooper, and P. M. Sutcliffe, Phys. Rev. Lett. 88, 080401 (2002).

[54] C. M. Savage and J. Ruostekoski, Phys. Rev. Lett. 91, 010403 (2003).

[55] J. Ruostekoski, Phys. Rev. A 70, 041601 (2004).

[56] T. Kawakami, T. Mizushima, M. Nitta, and K. Machida, Phys. Rev. Lett. 109, 015301 (2012).

[57] T. Isoshima, M. Nakahara, T. Ohmi, and K. Machida, Phys. Rev. A 61, 063610 (2000). 
[58] A. E. Leanhardt, A. Görlitz, A. P. Chikkatur, D. Kielpinski, Y. Shin, D. E. Pritchard, and W. Ketterle, Phys. Rev. Lett. 89, 190403 (2002).

[59] Y. Shin, M. Saba, M. Vengalattore, T. A. Pasquini, C. Sanner, A. E. Leanhardt, M. Prentiss, D. E. Pritchard, and W. Ketterle, Phys. Rev. Lett. 93, 160406 (2004).

[60] H. Mäkelä and K.-A. Suominen, Phys. Rev. A 75, 033610 (2007).

[61] S. Knoop, T. Schuster, R. Scelle, A. Trautmann, J. Appmeier, M. K. Oberthaler, E. Tiesinga, and E. Tiemann, Phys. Rev. A 83, 042704 (2011).

[62] J. Ruostekoski and Z. Dutton, Phys. Rev. A 76, 063607 (2007).
[63] E. G. M. van Kempen, S. J. J. M. F. Kokkelmans, D. J. Heinzen, and B. J. Verhaar, Phys. Rev. Lett. 88, 093201 (2002).

[64] In a strongly magnetized condensate, it becomes energetically favorable to empty $\zeta_{-}$, stabilizing the coreless vortex as an effective two-component vortex.

[65] M. R. Matthews, B. P. Anderson, P. C. Haljan, D. S. Hall, C. E. Wieman, and E. A. Cornell, Phys. Rev. Lett. 83, 2498 (1999).

[66] M. F. Andersen, C. Ryu, P. Cladé, V. Natarajan, A. Vaziri, K. Helmerson, and W. D. Phillips, Phys. Rev. Lett. 97, 170406 (2006). 